\title{
SIMULTANEOUS ITERATION BY ENTIRE OR RATIONAL FUNCTIONS AND THEIR INVERSES
}

\author{
I. N. BAKER and ZALMAN RUBINSTEIN
}

(Received 25 February 1980)

Communicated by E. Strzelecki

\begin{abstract}
For a non-constant entire or rational function $f$ normalized by $f(0)=0, f^{\prime}(0)=1, f^{\prime \prime}(0) \neq 0$, which is not a Möbius transformation, the existence of a sequence $\left\{z_{n}\right\}_{n=-\infty}^{n=+\infty}$ is established which has the properties $f\left(z_{n}\right)=z_{n+1}, \lim _{n \rightarrow \infty} z_{n}=\lim _{n \rightarrow-\infty} z_{n}=0$. The result certainly implies $f(0)=0,\left|f^{\prime}(0)\right|=$ 1 , so these conditions cannot be omitted. The condition $f^{\prime \prime}(0) \neq 0$ can be replaced by $f^{(k)}(0) \neq 0$ for some $k \geqslant 2$.
\end{abstract}

1980 Mathematics subject classification (Amer. Math. Soc.): primary 30 D 05; secondary 39 B 10.

\section{Introduction}

The theory of fixpoints and iterations of functions is of continuing interest both from the theoretical and numerical points of view. For example the volume [7] is entirely devoted to this topic. Iterations of entire functions were first studied seriously in [5] and more recently for example in [1], [6, chapter 2] and [8]. Iteration of polynomials and rational functions was treated in [4] with occasional more recent contributions such as [3].

Although iteration of the inverse functions of polynomials is discussed in the fundamental paper [4], the results seem to be relatively unknown. In the present paper some of these results are used to solve a problem which was raised recently by A. Shields and C. Pearcy (oral communication). They asked whether there exists a polynomial $f$ and an infinite sequence $\left\{z_{n}\right\}_{n=-\infty}^{\infty}$ of complex numbers such that $\lim _{n \rightarrow \infty} z_{n}=\lim _{n \rightarrow-\infty} z_{n}=0$ and $z_{n+1}=f\left(z_{n}\right)$ for all $n$.

We give a positive solution to this problem not only for polynomials but for entire or rational functions also. The second author first found the example

C Copyright Australian Mathematical Society 1983 
$f(z)=z+z^{2}$ by elementary arguments. This example is now included in the more general:

THEOREM. Let $f$ be a non-constant entire or rational function which is not a Moebius transformation. Suppose that $f(0)=0, f^{\prime}(0)=1$ and $f^{\prime \prime}(0) \neq 0$. Then there exists a sequence $\left\{z_{n}\right\}_{n=-\infty}^{\infty}$ of complex numbers such that

(i) $f\left(z_{n}\right)=z_{n+1}$ and

(ii) $\lim _{n \rightarrow+\infty} z_{n}=\lim _{n \rightarrow-\infty} z_{n}=0$.

Remark. The condition $f^{\prime \prime}(0) \neq 0$ is not essential and can be replaced by $f^{(k)}(0) \neq 0$ for some $k$ at the cost of complicating the description required for the proof. Without loss of generality we may assume that $f^{\prime \prime}(0)>0$ since a scale change $z_{n} \rightarrow t_{n}=\lambda^{-1} z_{n}, \lambda$ constant, replaces $f(t)$ by $\lambda^{-1} f(\lambda t)$. Denote by $f^{n}$ the $n$th iterate of $f, n \geqslant 0$, and let $\mathscr{F}=\mathscr{F}(f)$ be the set of those points $z$ in whose neighborhood the sequence $\left\{f^{n}\right\}$ is not a normal family. It was proved by Fatou in $[4,5]$ that for functions $f$ which are entire or rational but not Möbius transformations:

I. $\mathscr{F}(f)$ is a non-empty perfect set.

II. Given any $\beta \in \mathscr{F}$ then if $z$ is not one of the at most two exceptional values which depend only on $f$ and not on $\beta$, there is a sequence of integers $n_{k}$ and a sequence of complex numbers $z_{k}$ such that

$$
n_{k} \rightarrow \infty, z_{k} \rightarrow \beta, \quad f^{n_{k}}\left(z_{k}\right)=z .
$$

Note also that under the assumptions of the theorem $0 \in \mathscr{F}(f)$. Indeed we have $f^{n}(z)=z+n a_{2} z^{2}+\cdots, n \geqslant 0$, valid in a neighborhood of the origin. If a sequence $f^{n_{k}}$ is uniformly convergent to $g$ in a neighborhood of 0 we must have $g(0)=0$ so that $g$ is analytic at 0 and $\left[f^{n_{k}}(z)\right]^{\prime \prime} \rightarrow g^{\prime \prime}(z)$ at $z=0$, which conflicts with $n_{k} a_{2} \rightarrow \infty$.

Note also the following

LEMMA [2, Lemma 9, case $m=1$ ]. If $g(z)=z+a_{2} z^{2}+\cdots, a_{2}=\sigma e^{i \alpha}, \sigma>0$, $\alpha$ real, is analytic at $z=0$, then for a given $\theta$ with $0<\theta<\pi / 2$ and for all sufficiently small $\rho>0$ the iterates $g^{n}$ of $g$ are defined in

$$
D(\theta, \rho)=\{z: 0<|z|<\rho,-\gamma+\theta-\pi<\arg z<-\gamma-\theta+\pi\},
$$

where $\gamma=\alpha+\pi$. Further, $g^{n}(z) \rightarrow 0$ locally uniformly in $D(\theta, \rho)$. This means that for $z \in D(\theta, \rho)$ all the values $g^{n}(z)$ lie in $D(\theta, \rho)$. It turns out that the $g^{n}(z)$ approach 0 from the direction $\arg z=-\gamma$.

Proof OF THE THEOREM. By assumption the expansion of $f$ at 0 is $f(z)=z+$ $\sigma z^{2}+\cdots, \sigma>0$. Choose a fixed $\theta$ in $0<\theta<\pi / 2$ and apply the lemma with 
$\alpha=0$. There is $\rho>0$ such that in

$$
D(\theta, \rho)=\{z: 0<|z|<\rho, \theta<\arg z<2 \pi-\theta\}
$$

we have $f^{n}(z) \rightarrow 0$ locally uniformly. Thus $D(\rho, \theta)$ belongs to the complement of $\mathscr{F}(f)$.

The inverse function $f^{-1}$ has a branch $F$ whose expansion near 0 is

$$
F(z)=z-\boldsymbol{\sigma} z^{2}+\cdots,
$$

which converges for say $|z|<\delta$. Applying the lemma to $F$ with $\alpha=\pi$ shows that for sufficiently small positive $\rho^{\prime}$ the iterates $F^{n}, n \geqslant 1$ of $F$ are defined in

$$
D^{\prime}\left(\theta, \rho^{\prime}\right)=\left\{z: 0<|z|<\rho^{\prime}, \theta-\pi<\arg z<\pi-\theta\right\} .
$$

Again $F^{n}(z) \rightarrow 0$ as $n \rightarrow \infty$.

Take any $z_{0}$ in $D(\theta, \rho)$ which is not exceptional in the sense of II. Since $0 \in \mathscr{F}$ and $\mathscr{F}$ is perfect there are points in $\mathscr{F}-\{0\}$ arbitrarily near 0 and these points are not in $D(\theta, \rho)$. Thus every $D^{\prime}\left(\theta, \rho^{\prime}\right)$ must contain such points. By II there is therefore an integer $k>0$ and a point $z_{-k}$ in $D^{\prime}\left(\theta, \rho^{\prime}\right)$ such that $f^{k}\left(z_{-k}\right)=z_{0}$. Put

$$
z_{n}= \begin{cases}f^{m}\left(z_{-k}\right) & \text { if } n \geqslant-k \text { so that } m=n+k \geqslant 0 . \\ F^{m}\left(z_{-k}\right) & \text { if } n<-k \text { so that } m=-(n+k) \geqslant 0 .\end{cases}
$$

In particular $z_{0}=f^{k}\left(z_{-k}\right)$ which agrees with our earlier notation. It is immediate that $f\left(z_{n}\right)=z_{n+1}$ for all integers $n$. Further

$$
\begin{aligned}
& \lim _{n \rightarrow-\infty} z_{n}=\lim _{n \rightarrow \infty} F^{m}\left(z_{-k}\right)=0 \quad \text { since } z_{-k} \in D^{\prime}\left(\theta, \rho^{\prime}\right), \\
& \lim _{n \rightarrow \infty} z_{n}=\lim _{m \rightarrow \infty} f^{n+k}\left(z_{-k}\right)=\lim _{n \rightarrow \infty} f^{n}\left(z_{0}\right)=0 \text { since } z_{0} \in D(\theta, \rho) .
\end{aligned}
$$

\section{References}

[1] I. N. Baker, 'The existence of fixpoints of entire functions', Math. Z. 73 (1960), 280-284.

[2] I. N. Baker, 'Permutable power series and regular iteration', J. Austral. Math. Soc. 2 (1962), 265-294.

[3] I. N. Baker, 'Fixpoints of polynomials and rational functions', J. London Math. Soc. 39 (1964), 615-622.

[4] P. Fatou, 'Sur les équations fonctionelles', Bull. Soc. Math. France 47 (1919), 161-271; 48 (1920), 33-94, 208-314.

[5] P. Fatou, 'Sur l'itération des fonctions transcendantes entières', Acta Math. 47 (1926), 337-370.

[6] W. K. Hayman, Meromorphic functions (Oxford University Press, 1964). 
[7] Stepan Karamardian, Ed., Fixed points algorithms and applications (Academic Press, 1977).

[8] P. C. Rosenbloom, 'The fixpoints of entire functions', Meddelanden Lunds Univ. Mat. Sem. Suppl. Band M. Riesz (1952), 186-192.

Department of Mathematics

University of Michigan

Imperial College

Ann Arbor, Michigan 48109

London S.W. 7, U.K.

University of Haifa

Haifa, Israel 